\title{
Coulisses
}

Revue de théâtre

6 | Printemps 1992

Varia

\section{Britannicus, décor et scénographie}

\author{
Gisèle G. Holtzer
}

\section{OpenEdition}

Journals

Édition électronique

URL : http://journals.openedition.org/coulisses/1962

DOI : 10.4000/coulisses.1962

ISSN : 2546-9460

\section{Éditeur}

Presses universitaires de Franche-Comté

Édition imprimée

Date de publication : 1 juin 1992

ISSN : 1150-594X

\section{Référence électronique}

Gisèle G. Holtzer, «Britannicus, décor et scénographie », Coulisses [En ligne], 6 | Printemps 1992, mis en ligne le 15 mars 2019, consulté le 22 octobre 2019. URL : http://journals.openedition.org/coulisses/ 1962 ; DOI : 10.4000/coulisses.1962

Ce document a été généré automatiquement le 22 octobre 2019

Coulisses 


\section{Britannicus, décor et scénographie}

Gisèle G. Holtzer

L'art du décor au XVIIème siècle 
1 Racine donne au début de sa pièce les quelques indications scéniques suivantes : « La scène est à Rome, dans une chambre du palais de Néron ». Il n'est pas d'usage à l'époque que l'auteur apporte des précisions écrites sur le décor, les costumes, les accessoires. Le texte est pour le décorateur la principale source exploitable, les acteurs collaborant souvent à la liste des objets nécessaires. On connaît par le mémoire du décorateur de Britannicus, Michel Laurent, le décor des représentations qui eurent lieu à l'Hôtel de Bourgogne en 1669. La description tient en une brève énumération : "Le théâtre est un palais à volonté. Il faut 2 portes, 2 fauteuils pour le $4^{\text {ème }}$ acte ; des rideaux ». Les éléments du décor sont tous appelés par le texte :

- La porte de Néron : «La mère de César veille seule à sa porte » $(\mathrm{I}, 1)$

- La porte par où entre Junie : « On ouvre, la voici» (II, 2)

- Les rideaux, mur qui voit et écoute derrière lequel Néron observe Junie et Britannicus : « Caché près de ces lieux, je vous verrai, Madame » (II, 3).

Quant aux fauteuils, ils sont prévus pour la grande scène entre Agrippine et Néron $(I V, 2)$. Racine précise ce détail au début de l'acte IV : « Agrippine, s'asseyant ».

3 Vers 1660 et quel que soit le lieu où se situe la pièce, la tragédie classique se joue dans un décor quasi permanent que le public retrouve à chaque représentation nouvelle. C'est le palais "à volonté ", selon la formule des décorateurs, peint sur des toiles de fond avec un effet de perspective ; le palais à colonnes (Andromaque) ou voûté (Phèdre), s'ouvre en arrière-plan sur une cour ou un arc de triomphe, sur la mer et des vaisseaux selon le cas. Il existe quelques variantes d'une pièce à l'autre qui évitent la monotonie : arcades, statues, verdure. Il est clair que dans la tragédie, le décor est un cadre qui ne doit pas s'imposer au spectateur mais être oublié dès la première réplique. Le spectaculaire et le merveilleux ont leur place ailleurs, dans les pièces à machines, les comédies-ballets et l'opéra. De plus, la conception du décor n'obéit à aucun réel souci de vérité historique. La couleur locale se borne par exemple à un "salon à la turque » (Bajazet). En fait, le lieu de la tragédie est reproduction à peine stylisée de l'espace de l'époque. Le palais est peint sur le modèle de ceux du XVII ème siècle et, si l'on ajoute que les costumes de scène - exception faite pour les pièces inspirées de l'histoire romaine ne se différencient pas des habits de Cour, on voit que le lieu de la représentation est fait de signes visuels lisibles, évidents, reflétant la réalité mondaine du temps, dans une interpénétration du présent et du passé.

Ces faits suggèrent l'idée d'une esthétique théâtrale où le visuel n'est pas un constituant premier du spectacle. Plus précisément, le visuel n'est pas dans la réalité sensible. Il est dans le discours qui exprime, évoque, décrit. Le théâtre, écrit - dans une formulation un peu paradoxale - l'abbé d'Aubignac, grand théoricien du spectacle dramatique, est « un Lieu où on regarde ce qui s'y fait, et non pas où l'on Ecoute ce qui s'y dit. Aussi est-il vrai que les discours qui s'y font, doivent être comme des Actions de ceux qu'on y fait paraître ; car là Parler, c'est Agir (...). En un mot, les Discours ne sont au théâtre que les accessoires de l'Action ». C'est donc le discours qui fait voir: spectacle des passions exprimées (amour, haine, douleur...), événements et actions retracés par les narrations. L'enlèvement de Junie est l'un des grands tableaux 
raciniens (II, 2), revécu par Néron en une scène onirique où la description, filtrée par sa subjectivité, est composée sur les contrastes: ombre/lumière, cris/silence, impassibilité/tristesse, dureté (des soldats)/vulnérabilité (de la victime). On sait l'importance de la thématique du regard chez Racine, regard qui voit et donne à voir dans les récits, regard qui parle : «J'entendrai des regards que vous croirez muets » dit Néron à Junie (II, 3).

5 L'art du dramaturge sera d'exceller, par la force des vers, dans le discours qui suggère, stimule l'imagination du spectateur, touche sa sensibilité. Cela explique que chez l'acteur tragique le dire soit plus travaillé que l'expression corporelle. Le plaisir du spectateur est l'écoute de la parole dans son pouvoir d'évocation, ce qui suppose un mode de participation très différent de celui du spectacle contemporain.

\section{Scénographie :}

6 La scénographie, qui a pris naissance à la fin du XIX ${ }^{\text {ème }}$ siècle, occupe une place de plus en plus importante dans le théâtre contemporain. Récusant le terme trop limitatif de " décorateur ", le scénographe se veut une sorte de co-auteur du spectacle. "J'estime, dit Y. Kokkos qui assura la scénographie du Britannicus monté par Vitez en 1981, que le scénographe ne produit pas seulement l'image finale, mais il participe à l'intégralité du processus qui mène vers la conception qui régit une mise en scène (...). Notre participation très poussée à la réalisation d'un spectacle ne peut pas être désignée à l'aide du mot «décorateur ». Ce qui me gêne dans ce mot, c'est l'idée de décoration, d'ornement, d'emballage ». Le scénographe est l'organisateur d'un point de vue. La mise en espace, la création d'un lieu est toujours regard sur l'œuvre, interprétation, et en cela la scénographie est composante, avec la mise en scène, de la dramaturgie de la pièce.

\section{Le Britannicus de Gildas Bourdet (1980) :}

7 Comme pour la plupart des spectacles de la Salamandre, G. Bourdet a assuré à la fois la mise en scène et la scénographie de la tragédie de Racine. Le décor situe l'action au XVII ème siècle et présente, avec un grand souci de fidélité et de réalisme, un fastueux salon versaillais. Sur une console, un buste d'empereur romain est le seul indice de romanité mais il a sa place naturelle dans l'espace de Louis XIV qui se veut héritier des Césars. Des chaises rangées le long du mur évoquent l'antichambre qui jouxte le lieu du pouvoir dont l'accès est la porte, frontière majestueuse et infranchissable encadrée par d'imposants tableaux allégoriques à la gloire du Maître. L'image renvoyée par ce décorreconstitution est celle d'un lieu froid et dominateur qui surplombe les personnages. Dans cet espace, symbole d'un ordre du monde, la place de chacun est pré-fixée par le désir du Prince. «Dans le décor de Britannicus, dit Gildas Bourdet, il n'y avait pas du tout de vivant. Au contraire j'ai cherché le lieu le moins naturel possible, le moins aménagé pour la vie (...). Toute harmonie entre la réalité du corps dans son intimité, dans ses faiblesses, et ce lieu était exclue, car le corps dont Versailles représente le décor n'est pas un corps humain mais le corps du Roi, le corps du pouvoir ». 


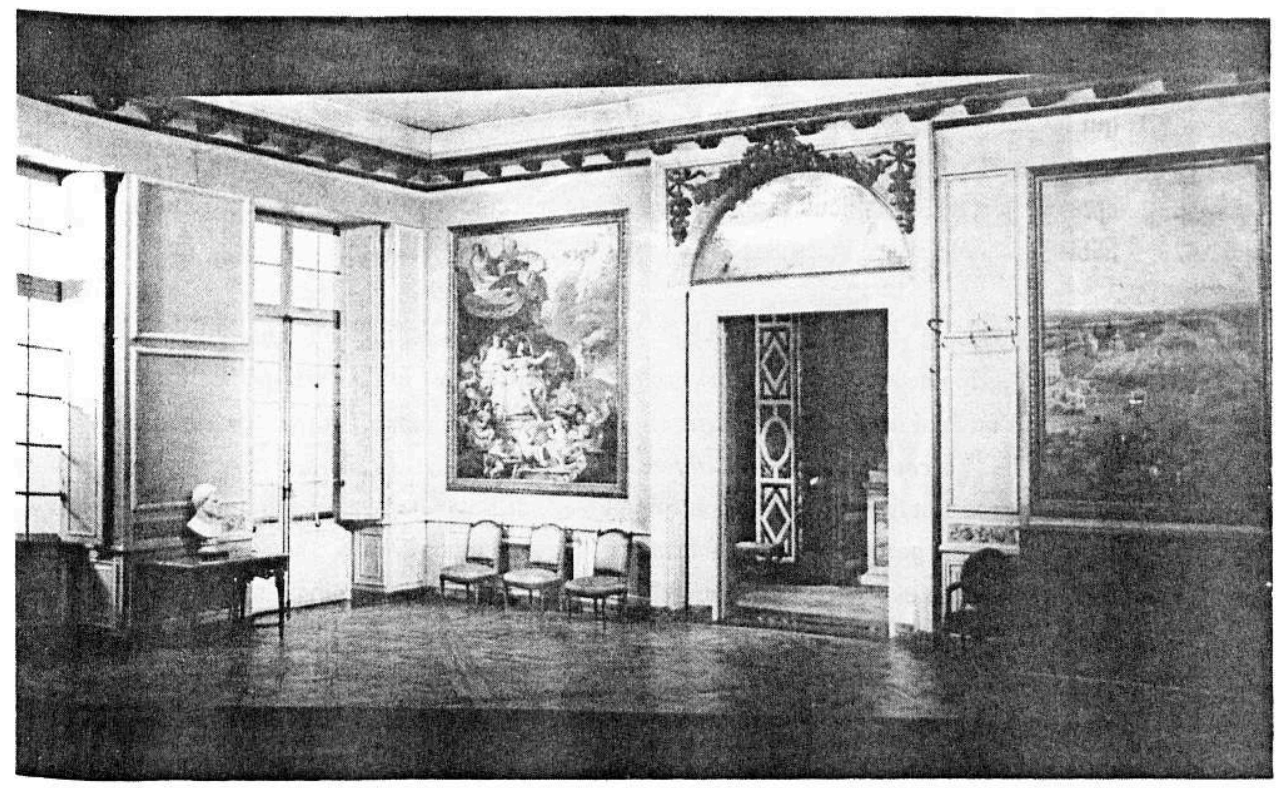

\section{Le Britannicus d'Alain Françon (1992) :}

Britannicus a été donné du 12 au 15 février 1992, au Théâtre Municipal de Besançon. Il s'agit d'une production théâtre du Huitième, Centre Dramatique National de Lyon que dirige Alain Françon. Le spectacle a également été présenté au Théâtre des Amandiers (Nanterre) du 7 janvier au 2 février 92.

8 Ce qui semble avoir intéressé Alain Françon, c'est - à l'intérieur d'un échiquier politique aux puissants enjeux de position (la situation de la Reine-Roi Agrippine est fragile, le pouvoir lui échappe, ses appuis doivent être rapidement décidés et placés) le passage de Néron de l'état d'adolescent en révolte contre la tutelle maternelle à l'état affirmé de «maître du monde ». Dans sa pièce, Racine s'était proposé de montrer en Néron «un monstre naissant»; Alain Françon, plutôt que le monstre, choisit la naissance, plus exactement la « renaissance de Néron qui, se défaisant de toutes ses chaînes - mère, femme, frère, gouverneur - refusant tout héritage historique, s'accouche une deuxième fois dans la souffrance » (A. Françon, Notes, août 91).

Loin du personnage hystérique confinant au cas pathologique que nombre de mises en scène nous ont présenté, Françon montre un Néron solaire, dont la blondeur répond à celle de Junie, frêle dans un habit noir aux parements blancs (référence claire à Hamlet et au Prince de Hombourg) qui lui donne une aura romantique. En vingt-quatre heures, durée réglementaire de la tragédie classique, Néron effectuera sa propre libération, signifiée par la transformation de son apparence: après la mort de Britannicus, la pourpre impériale et la couronne des Césars remplacent le sombre habit de l'adolescence incertaine.

10 Face à ce fils-empereur en devenir, Nada Strancar, chevelure de feu et robe rouge carmin, est une Agrippine violente aux furieuses frustrations. C'est elle le vrai personnage tragique, marqué d'un fatal destin : elle mourra, elle le sait, des astrologues le lui ont dit il y a longtemps «Le coup qu'on m'a prédit va tomber sur ma tête » $(\mathrm{V}, 7)$. 
Dans la nuit finissante, Agrippine fait le siège de l'appartement de son fils, couchée devant sa porte. A cette porte qu'elle ne peut franchir s'arrête son pouvoir. La position couchée est à la fois aveu de faiblesse (l'accès du lieu est interdit) et de ferme détermination (pour sortir, Néron devra lui passer sur le corps et l'entendre). De ce corps allongé, auquel l'obscurité donne des contours indécis, sort une voix puissante à la tonalité grave (connotation de masculinité). Avec Nada Strancar, l'alexandrin sonne fort, passant de l'explosion violente de l'autorité perdue au murmure éclatant de la fureur rentrée.

Hélène Alexandridis (Junie) et Clovis Cornillac (Britannicus)

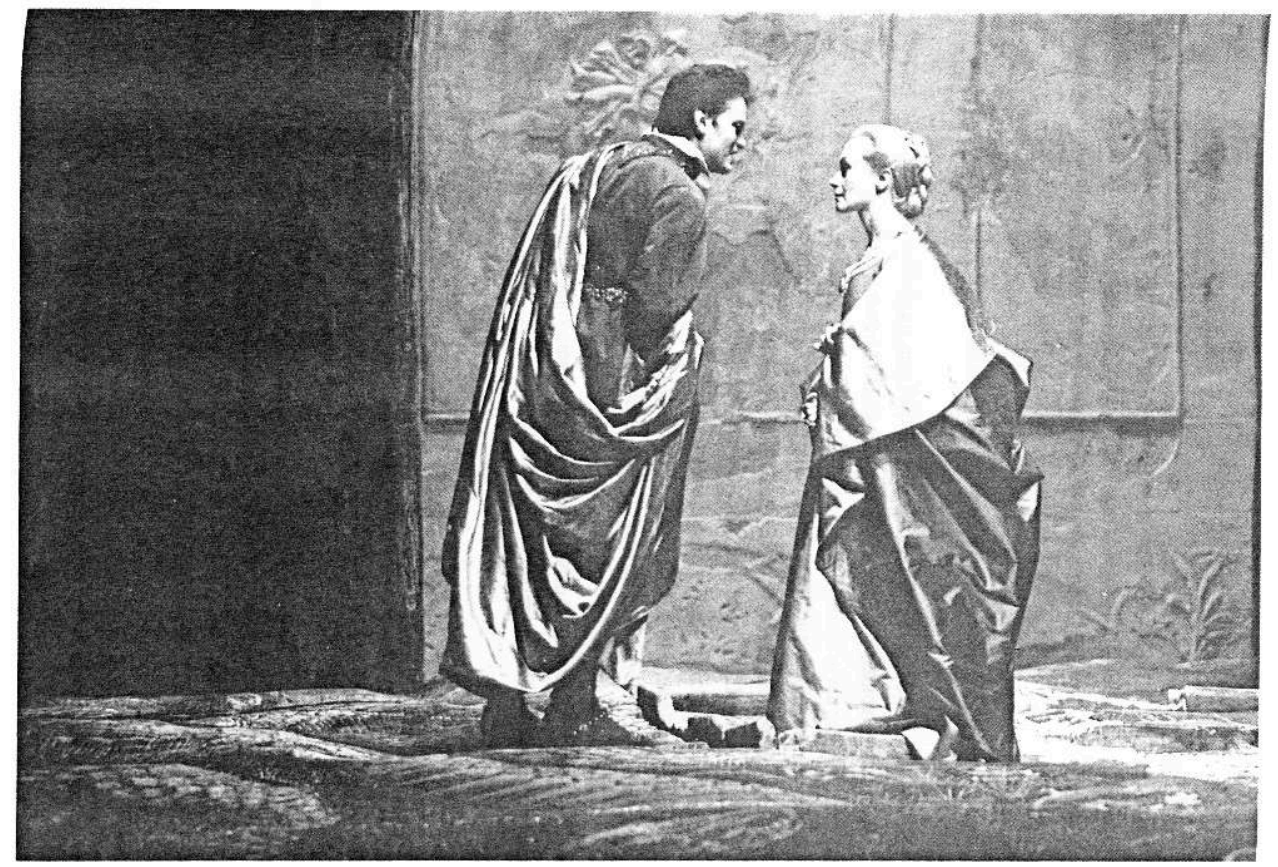

Photo Christian Ganet

\section{Notes éparses}

Un plateau bombé fait de gigantesques reptiles verdâtres, déroulant leurs anneaux sur toute la surface de la scène. Plus que les nœuds reptiliens de rapports familiaux venimeux, les serpents m'évoquent l'image de faux enlacements, de fourbes enveloppements. J'y vois la transposition visuelle des paroles de Néron (IV, 3) : «J'embrasse mon rival, mais c'est pour l'étouffer ».

Beauté des drapés: le vêtement de Junie, tissu sec au tombé un peu raide comme l'inflexibilité du personnage. Elle ne cède rien et, avec elle, Néron a perdu.

14 Jeu sur l'opposition verticalité/horizontalité des corps: Agrippine couchée devant la porte de Néron (I, 1), étendue sur le sol à côté de son fils (IV,2). Verticalité de l'affrontement physique Britannicus-Néron (III, 8), en écho au duel des répliques verbales :

BRITANNICUS. - Ainsi Néron commence à ne plus se forcer.

NÉRON. - Néron de vos discours commence à se lasser. 
15 Verticalité et éloignement: Junie et Néron aux deux extrémités d'une diagonale imaginaire, deux personnalités irréductiblement étrangères l'une à l'autre. 